\title{
EchoGéo
}

$14 \mid 2010$

Afrique, 50 ans d'indépendance : Dynamiques spatiales, identités, circulations

\section{Inventaire des cartes climatiques conservées à la cartothèque de l' IGN}

Bernadette Joseph

\section{(2) OpenEdition}

\section{Journals}

Édition électronique

URL : https://journals.openedition.org/echogeo/12242

DOI : $10.4000 /$ echogeo. 12242

ISSN : 1963-1197

Éditeur

Pôle de recherche pour l'organisation et la diffusion de l'information géographique (CNRS UMR 8586)

Référence électronique

Bernadette Joseph, « Inventaire des cartes climatiques conservées à la cartothèque de I' IGN », EchoGéo [En ligne], 14 | 2010, mis en ligne le 16 décembre 2010, consulté le 01 août 2021. URL : http:// journals.openedition.org/echogeo/12242 ; DOI : https://doi.org/10.4000/echogeo.12242

Ce document a été généré automatiquement le 1 août 2021

EchoGéo est mis à disposition selon les termes de la licence Creative Commons Attribution - Pas d'Utilisation Commerciale - Pas de Modification 4.0 International (CC BY-NC-ND) 


\section{Inventaire des cartes climatiques conservées à la cartothèque de $\mathrm{l}^{\prime}$ IGN}

Bernadette Joseph

\section{Afrique de l'Ouest et Centrale}

1 Précipitations moyennes annuelles 1951-1989.- 1 : 6000 000.- ORSTOM, 1996.

\section{Afrique de l' Est}

Possibilités de chutes annuelles de pluies sur 4 ou 5 ans.- 1 : 4000 000.- 1961.

\section{Afrique}

3 Carte pluviométrique.- $1: 5000$ 000.- DOS, 1958.- 6 feuilles.

\section{Afrique (Nord Ouest)}

$4 \quad 1$ :7500 000.- IGN ; Météorologie Nationale, 1975.

\section{Afrique AEF}

5 Carte pluviométrique sous la direction de E. Bannelier.- 1 : 10000 000.- s.n., 1935.

\section{Algérie}

6 Plaine de la Mitidja : isohyètes mensuelles février - juillet.- IGN, 1970.- 1 : 100 000.- 6 feuilles. 
7 Carte des précipitations / H. Gaussen.- 1 : 500 000.- Gouvernement général de l'Algérie, 1966.- 4 feuilles.

8 Carte des précipitations annuelles, mensuelles, fréquentielles de l'Algérie septentrionale.- 1 : 1000 000.- s.n., 1972.- 5 feuilles.

9 Carte pluviométrique : moyenne annuelle 1913-1963 / Chaumont, Paquin.- 1 : $500000 .-$ Université d' Alger, Faculté de sciences, 1971.- Feuilles: Oran, El Asmar, Alger, Constantine.

\section{Allemagne}

Carte de distribution des pluies entre 1891 et 1920.- 1 : 1000 000.- s.n., 1936 (éd. allemande).

\section{Asie}

11 Climatic regions map of Asia and the Far East.- 1: 5000 000.- Royal Survey Dépt., 1973.4 feuilles.

\section{Australie}

Climatic regions.- 1: 6000000 .- National Mapping Authority, 1954.

Median annual rainfall.- 1: 10000 000.- National Mapping Authority, 1980.

Monthly temperatures.- 1: 30000 000.- National Mapping Authority , 1983.

5 Climate of capital cities.- 1: 500 000.- National Mapping Authority,1984.

\section{Brésil}

Hydrological studies d'upper Paraguay river basin: pluviometric network, evaporemetric network.- 1: 2000 000.- PNUD UNESCO, 1974. feuilles. Isohyètes for the period octobre1969 - septembre 1970.- 1974.

Isohyètes for the period octobre1970 - septembre 971.- 1974.

\section{Cameroun}

Hauteurs moyennes annuelles de pluies en mètres.- $1: 4500$ 000.- Brazzaville : Service Géographique, 1960.

Cameroun.- 1 : 12000 000.- IGN, Service de la Météorologie du Cameroun, 1947.

Carte physiologique et climatique.- éd. allemande, s.d.

\footnotetext{
Carte des pluviosités.- 1 : 20000 000.- éd. allemande, s.d.
} 


\section{Canada} Association franco chinoise de la recherche scientifique, 1998.- 3 feuilles.

\section{Colombie}

\section{Cote d' Ivoire}

\section{Espagne -Portugal}

Carte climatique de la Péninsule Ibérique.- 1000 000.- CNRS, 1969.-1 feuille + notice.

\section{France}

Carte climatique détaillée de la France sous la direction de Charles Péguy.- $1: 250000$.Ed Ophyrs, 1968. (Les feuilles de la " Carte climatique détaillée de la France", sont répertoriées dans le SUDOC, et localisées dans les bibliothèques universitaires, seulement).

37 Carte des précipitations totales pour l'année 1971 sur le Bassin Seine- Normandie.- 1: 500 000.- Université de Caen , faculté lettres sciences humaines, 1971.

38 Carte de pluviosité annuelle du sud ouest de la France, Pyrénées, Alpes, bassin du Rhône et Corse, sous la direction de Gaussen.- 1 : 500 000.- SGA, 1936. 

de France en Indonésie, 1978.

\section{Laos}

\section{Liban}

Carte pluviométrique.- 1 : 200 000.- IGN, 1954.

\section{Madagascar}

Madagasikara; Ny Sarintanin; Ny Toetany.- 1 : 125 0000.- Ed FTM,1983.

Carte bioclimatique de Madagascar.- 1 : 2000 000.- ORSTOM, 1973.

\section{Maroc}

Carte de précipitations moyennes annuelles ramenées à la période 1925-1950 - 1: 500 000.- IGN ; Ministère de l' agriculture et des forets, 1957.- 6 feuilles.

Moyenne annuelle des précipitations 1926-1940.- 1:1500000.- IGN; Institut scientifique Chérifien. 


\section{Mauritanie}

Carte des pluies en 1928.- 1:2 000 000.- Service géographique de l'AOF, 1929.

\section{Mexique}

Carta de climas.- 1: 500 000.- Instituto de geografia e la presidencia, 1970.- Feuilles : Quereato 14QIII Chifma Hua 73RIII. Matamoros y Reynosa 14RVIII 14RVI Chifma Hua 73RIII. Matamoros y Reynosa 14RVIII 14RVI.

\section{Monde}

Stations of desertification, climate aridity, scheme of aridity and drought probability.$1: 25000$ 000.- UNESCO ; FAO, 1977 (Conference of desertification).

Carte des environnements du Monde pendant les deux derniers extrêmes climatiques.1: 25000 000.- IGN ; Commission de la carte géologique du Monde, 1999.- 2 feuilles : dernier maximum glaciaire ; carte de l'optimum holocène.

Climats et zones de végétation.- 1 : 25000 000.- Geodatik Institue of Kobenhaven, 1961.

Principaux groupes de régions arides dans le Monde.- 1:50 000 000.- IGN, 1963.

Zones et périodes de mauvais temps.- $1: 150000$ 000.- SHOM (Brest), 1961.

\section{Nouvelle Calédonie}

Carte des précipitations annuelles 1966.- 1 : 4000 000.- s.n.- 1 feuille + notice.

\section{Nouvelle Zélande}

Air temperatures annual 1985.- 1: 2000000 .- Dept of lands survey méteorological service, 1985.

Climatic regions.- 1: 2000 000.- Dept of lands survey méteorological service , 1982.

Climatologicals station.- 1: 2000 000.- Dept of lands survey méteorological service , 1983.

\section{Pologne}

Carte climatique.- 1: 15000 00.- Ed E. Romer, 1938 (ed. polonaise).

\section{Roumanie}

Précipitations atmosphériques.- 1: 1500 000.- Institutul de geologia si geografie al Academiei, 1954.- Feuille 16.

Répartition des températures de l'air ; moyennes annuelles 1896-1955.- 1 : 1500000 .Institutul de geologia si geografie al Academiei, 1959.- Feuilles 12, 14, 15. 


\section{Thailande}

\section{Tunisie} 1900-1940.- 2 feuilles.

\section{RÉSUMÉS}

Cet inventaire présente la liste des cartes climatiques étrangères, conservées à la cartothèque de l' IGN. Ce fonds très riche en cartes thématiques n'est actuellement pas répertorié dans les catalogues SUDOC ou OPALE. Il n'est accessible qu'en interne sur fichiers papier. 